\title{
Dietary Cholesterol and Plasma Lipoprotein Profiles: Randomized Controlled Trials
}

\author{
John D. Griffin • Alice H. Lichtenstein
}

Published online: 18 October 2013

(C) Springer Science+Business Media New York 2013

\begin{abstract}
Early work suggested that dietary cholesterol increased plasma total cholesterol concentrations in humans. Given the relationship between elevated plasma cholesterol concentrations and cardiovascular disease risk, dietary guidelines have consistently recommended limiting food sources of cholesterol. Current intakes are approaching recommended levels. Recently there have been calls to reassess the importance of continuing to recommend limiting dietary cholesterol. Over the past 10 years, there have been a limited number of studies addressing this issue. Striking among these studies is the high degree of variability in background diet, study subject characteristics, and study design. Within the context of current levels of dietary cholesterol intake, the effect on plasma lipid concentrations, with primary interest in LDL cholesterol concentrations, is modest and appears to be limited to population subgroups. In these cases, restrictions in dietary cholesterol intake are likely warranted. The biological determinants of interindividual variability remain a relatively understudied area.
\end{abstract}

Keywords Dietary cholesterol · Plasma cholesterol · Low density lipoprotein cholesterol $\cdot$ High density lipoprotein cholesterol - Total cholesterol to high density lipoprotein cholesterol ratio $\cdot$ Eggs $\cdot$ Cardiovascular disease Cholesterol absorption

\section{Introduction}

Cardiovascular disease (CVD) is the leading cause of mortality in developed and developing countries [1]. Dyslipidemia, high low-density lipoprotein cholesterol (LDL-C) and triglyceride concentrations, and low high-density lipoprotein cholesterol (HDL-C) concentrations, are associated with elevated CVD risk [2]. There are many factors that impact on plasma lipoprotein profiles, including those that are non-modifiable (e.g., age, genetics) and modifiable (e.g., diet, physical activity). One that received considerable attention early in the elucidation phase of the diet/heart disease hypothesis is dietary cholesterol [3].

In humans, there is a linear relationship between dietary cholesterol up to about $1000 \mathrm{mg} /$ day and plasma cholesterol concentrations [4]. Most recommendations to reduce CVD risk include limiting dietary cholesterol to 200 or $300 \mathrm{mg}$ per day [2, 4-6]. Mean intakes in the U.S. are currently estimated to be $224 \mathrm{mg}$ and $333 \mathrm{mg}$ cholesterol per day for females and males, respectively. This review addresses the relationship between dietary cholesterol and plasma lipoprotein profiles, and is limited to randomized controlled trials published within the past 10 years.

\section{Cholesterol}

The cholesterol molecule has a steroid structure and hydrocarbon side chain. Technically, it is a sterol because it is composed of a steroid and alcohol. Cholesterol has multiple functions in the human body. It is a precursor for steroid hormones (gonadal and adrenal), vitamin D, and bile acids, and is a structural component of cell membranes and lipoprotein particles [7]. The substrate for cholesterol synthesis is acetyl CoA, an intermediate of fat, carbohydrate, and protein metabolism. 
The total body cholesterol pool reflects a balance between de novo synthesis, dietary intake, absorption efficiency, metabolism, enterohepatic recirculation, and excretion (either as cholesterol or bile acids). One of the primary tissues responsible for cholesterol homeostasis is the liver. Hepatic cholesterol is determined by a balance between LDL uptake from plasma and intracellular de novo synthesis. Liver cells have LDL receptors that are subject to feedback inhibition. These receptors recognize apoprotein (apo) B100, the protein component of the LDL particle, and apo E, an apoprotein component of chylomicron remnant, very low-density lipoprotein, and intermediate-density lipoprotein particles. After binding apo B100 or E present on the surface of lipoprotein particles, the LDL receptor and the particle are taken up into the cell by endocytosis. The receptor can be recycled; the lipoprotein particle is disassembled into its constituent components. The subsequent release of free cholesterol into the cytosol has three main effects: (1) inhibition of 3-hydroxy-3-methylglutaryl-CoA (HMG CoA) reductase activity, the rate-limiting enzyme in cholesterol biosynthesis, slowing the rate of de novo cholesterol synthesis; (2) increased activity of acyl CoA cholesterol acyltransferase (ACAT), the enzyme that esterifies intracellular cholesterol, yielding a more non-polar molecule that coalesces into intracellular lipid droplets; and (3) inhibition of LDL receptor synthesis, resulting in a decreased uptake rate of LDL from plasma. These mechanisms allow for a reciprocal relationship between plasma LDL cholesterol concentrations and hepatic de novo cholesterol synthesis [8].

The intestine plays an important role in whole-body cholesterol balance by regulating cholesterol absorption. Intestinal cholesterol is derived from dietary sources, sloughed cells from the intestinal lining, and direct secretion via bile [9]. The process by which cholesterol is absorbed is complex, involving a balance between transport into the enterocytes, facilitated by the Niemann-Pick C1-like 1 (NPC1L1) protein, and transport out of the enterocytes by ATP-binding cassette (ABC) $\mathrm{G} 5 / 8$ and possibly by an alternate pathway termed transintestinal cholesterol efflux. Free cholesterol associated with NPC1L1 is delivered to the endoplasmic reticulum, where the majority is esterified by ACAT and incorporation into chylomicron particles for subsequent secretion into the lymphatic system. The portion of intracellular cholesterol that remains unesterified forms a pool for potential resecretion into the intestinal lumen. Plant sterols (phytosterols) are structurally similar to cholesterol. They interfere with cholesterol absorption by displacing cholesterol from intestinal micelles. They are poor substrates for ACAT, hence a high proportion are resecreted into the intestinal lumen. Plant sterols have a low absorption efficiency, $1 \%$ to $3 \%$, compared to $50 \%$ to $60 \%$ for cholesterol. Another factor mediated by the intestine that impacts on the whole-body cholesterol pool is bile acids, a metabolic product of cholesterol. After secretion into the intestine, bile acids are recycled by an extremely efficient process of enterohepatic recirculation facilitated by apical $\mathrm{Na}$-dependent bile acid transporters. Any interference with this cycle will result in a net loss of bile acids in the stool, forcing the body to draw from the cholesterol pool to synthesize bile acids.

\section{Dietary Cholesterol and Plasma Cholesterol}

Early work suggested that dietary cholesterol increased plasma total cholesterol concentrations in humans [10], and this observation was confirmed by subsequent work [11-13]. Given the relationship between elevated plasma cholesterol concentrations and CVD risk, dietary guidelines have consistently recommended limiting food sources of cholesterol [2, 5, 14-19]. Recent reviews on the topic have questioned whether the relationship is still relevant within the context of current cholesterol intakes [20-22]. The issue remains unresolved. One issue that is difficult to factor into dietary cholesterol recommendations is individual responsiveness. It has been clearly shown that individuals vary considerably with respect to their response to dietary cholesterol [23]. Subsequent work has demonstrated that the distinction between hyperresponders or hyporesponders has a genetic basis, dependent on polymorphisms in the cholesterol transporters ABCG5/8 and NPC1L1, among others [24]. However, at this time, there is no simple way to determine whether an individual is a hyperresponder or hyporesponder to dietary cholesterol.

\section{Dietary Cholesterol}

Potential sources of dietary cholesterol are limited to animal foods: eggs, dairy products and meat. The majority of dietary cholesterol in the U.S. diet comes from eggs and egg mixed dishes (24.6\% of total cholesterol intake) [25]. Chicken and chicken mixed dishes (12.5\%) and beef, beef mixed dishes, and burgers $(11.0 \%)$ are the two next largest contributors. These are followed by regular cheese (4.2\%); sausage, franks, bacon, and ribs (3.9\%); fish and fish mixed dishes (3.4\%); grain-based desserts (3.3\%); dairy desserts $(3.2 \%)$; pasta and pasta dishes (3.1\%); pizza (2.9\%); Mexican mixed dishes (2.9\%); cold cuts $(2.7 \%)$; reduced-fat milk $(2.5 \%)$; pork and pork mixed dishes (2.3\%); and shrimp and shrimp mixed dishes (2.0\%). Together, these sources account for $85 \%$ of total dietary cholesterol.

\section{Literature Identification}

Studies that assessed the effect of dietary cholesterol on plasma lipoprotein profiles in humans were identified by 
electronic searches of the MEDLINE database using search criteria "cholesterol," " dietary" [MeSH Terms] or "cholesterol" [All Fields] and "dietary" [All Fields] or "dietary cholesterol" [All Fields] or "dietary" [All Fields] and "cholesterol" [All Fields] and "eggs" [MeSH Terms] OR "eggs" [All Fields] and "dietary cholesterol". Predetermined inclusion criteria were: (1) published between 3/1/2003 and 2/28/2013; (2) published in the English language; (3) conducted using human subjects; (4) included data for at least three of the following (not necessarily a primary analysis): total cholesterol (TC), LDL-C, HDL-C and triglyceride concentrations, and TC/ HDL-C ratio; (5) included an intervention which increased dietary cholesterol by a minimum of $200 \mathrm{mg}$ /day; and (6) held dietary fat type constant. Both crossover and parallel design studies were included. Reviews, case reports, and observational studies were not included.

\section{Results}

The electronic database searches resulted in 112 citations (Fig. 1). Seventeen studies met the inclusion criteria on the basis of abstract screening and 12 studies remained on the basis of full text screening (Table 1). Of the 12 studies, 7 controlled for the background diet [26-29, 30•, 31•, 32], and 5 studies did not control or did not specify whether they controlled for background diet [33-36, 37•]. In 3 of those 7 studies, subjects were instructed to modify their diets to promote weight loss [26-28], and 4 studies were specifically designed to maintain body weight $[29,30 \bullet, 31 \bullet, 32]$. In the vast majority of studies, eggs were used as the source of dietary cholesterol.

\section{Dietary Cholesterol Supplementation While Controlling for Background Diet}

\section{Dietary Cholesterol Supplementation with Weight Loss}

Using a parallel study design, Mutungi et al. assessed the effect of carbohydrate restriction and dietary cholesterol in 28 overweight or obese (BMI of $26-37 \mathrm{~kg} / \mathrm{m}^{2}$ ) male subjects aged 40-70 years [27]. Subjects were instructed to reduce energy intake by consuming a low-carbohydrate (10-15\% energy carbohydrate) diet and were randomly assigned to supplement this diet with 3 eggs per day ( $640 \mathrm{mg}$ cholesterol) or cholesterol-free egg substitute $(0 \mathrm{mg}$ cholesterol) which was provided to them for 12 weeks. The intervention resulted in significant reductions in body weight. Plasma LDL-C and triglyceride concentrations were similar between the two intervention groups, whereas HDL-C concentrations were higher in the egg-supplemented group.
Harman et al. randomly assigned 67 healthy volunteers, aged 18-55 years, to an energy-restricted diet (500-100 kcals less than baseline). In a 12-week parallel study design, subjects received either 2 eggs per day ( $438 \mathrm{mg}$ cholesterol) or no supplemental eggs [28]. At the end of the study period, both groups had lost a similar amount of body weight. The addition of eggs had no significant effect on plasma lipids.

Pearce et al. randomly assigned 65 individuals aged 2075 years with type 2 diabetes to consume a high-protein ( $30 \%$ of total energy) hypoenergetic diet (calories reduced $30 \%$ from habitual diet) with either 2 eggs per day (590 mg cholesterol per day) or 100 grams of lean protein (213 $\mathrm{mg}$ cholesterol per day) [26]. The hypoenergetic diet resulted in weight loss in both the high-cholesterol $(6.5 \mathrm{~kg}$ $\pm 3.2 \mathrm{~kg})$ and low-cholesterol $(5.3 \mathrm{~kg} \pm 3.6 \mathrm{~kg})$ groups. At the end of the 12-week period, plasma HDL-C concentrations were higher in the egg-fed group compared to the lean protein group, whereas there was no significant change in the other plasma lipids.

\section{Dietary Cholesterol Supplementation Without Weight Loss}

Using a parallel study design, Vislocky et al. assessed the effect of either $12 \mathrm{eggs} /$ week (469 $\mathrm{mg}$ cholesterol/day) or no supplemental eggs (159 mg cholesterol/day) on plasma lipid and apolipoprotein concentrations in 12 normocholesterolemic physically unfit subjects aged 18 to 30 years (men, $\mathrm{VO}_{2}$ peak $<45 \mathrm{~mL} / \mathrm{kg}^{-1}$ per $\mathrm{min}^{-1}$; and women, $<40 \mathrm{~mL} / \mathrm{kg}^{-1}$ per $\mathrm{min}^{-1}$ ) [29]. All diets were similar with regard to macronutrient content (60\% carbohydrate, $30 \%$ fat, $10 \%$ protein) and designed to maintain body weight. Subjects began the dietary intervention 2 weeks before starting an endurance training program and continued throughout the 6-week training period. Independent of cholesterol intake, endurance training significantly increased HDL-C and decreased LDL-C and apo-B concentrations, and increased plasma cholesterol ester transfer protein activity. Supplementation with an average of one egg per day had no significant effect on plasma lipids or the enzyme parameters assessed.

Knopp et al. studied three groups of individuals: insulin-sensitive (Insulin Sensitivity Index Score $<4.2 \times$ $1.0^{-4} \mathrm{~min}^{-1} \mu \mathrm{U} / \mathrm{mL}, \mathrm{n}=65$ ) (average age 49.4 years); insulin-resistant, normal body weight (Insulin Sensitivity Index Score $\geq 4.2 \times 1.0^{-4} \mathrm{~min}^{-1} \mu \mathrm{U} / \mathrm{mL} ; \mathrm{BMI}<27.5, \mathrm{n}=75$ ) (average age 55.0 years); and insulin-resistant, obese (Insulin Sensitivity Index Score $\geq 4.2 \times 1.0^{-4} \mathrm{~min}^{-1} \mu \mathrm{U} / \mathrm{mL}$; BMI $\geq 27.5, \mathrm{n}=57$ ) (average age 54.1 years) using a randomized crossover design [32]. Baseline plasma lipid concentrations ( 0 -egg-diet period) were not significantly different among the three groups. The dietary interventions were no supplemental eggs, 2 eggs per day (425 mg cholesterol) and 4 eggs per day (850 mg cholesterol). Each intervention period was 4 weeks, followed by a 4- 
Fig. 1 Flow diagram showing the assessment of intervention trials involving dietary cholesterol from 2003-2013

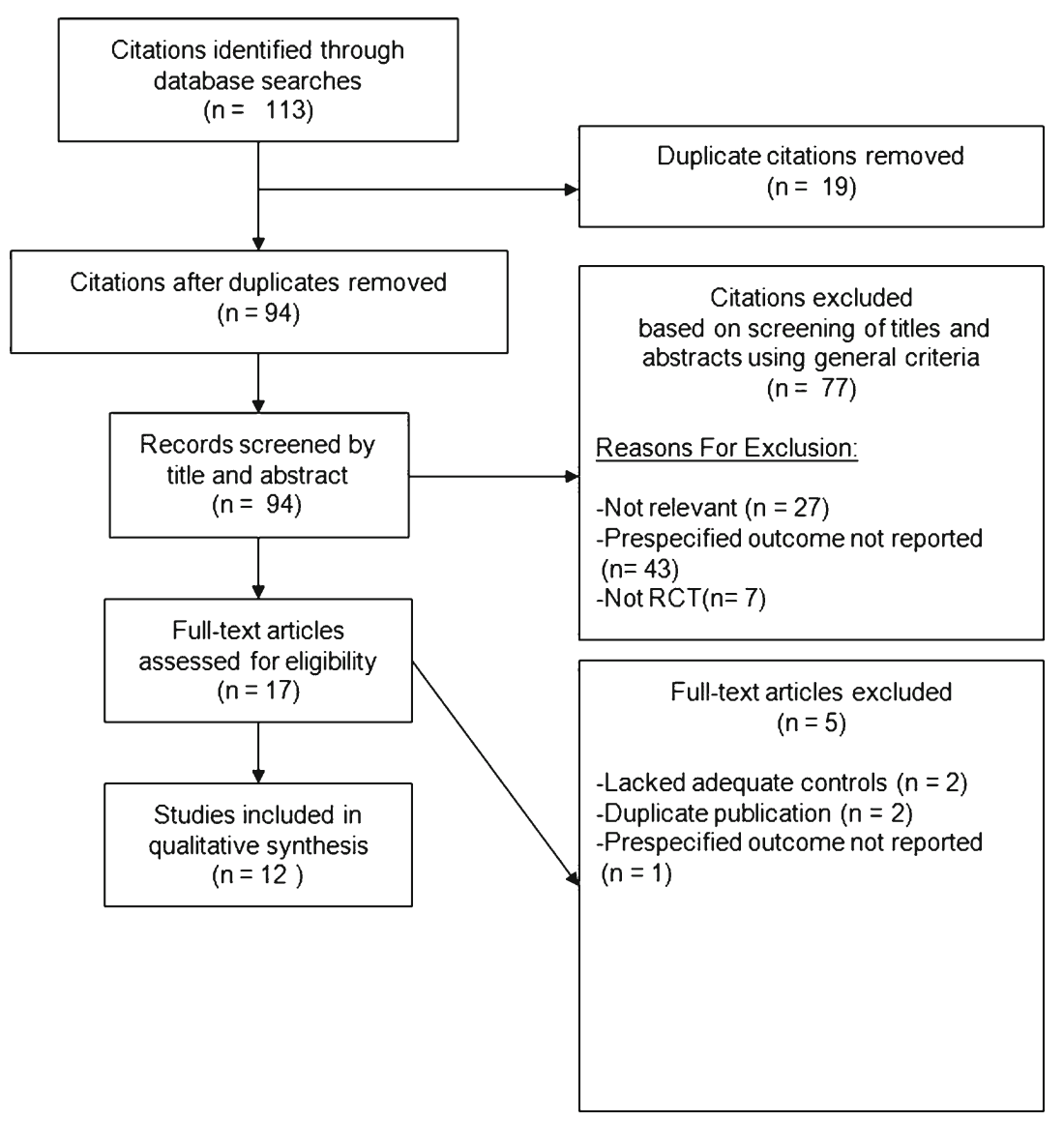

week washout period. During the intervention, the background diet was standardized by instructing subjects to adhere to the National Cholesterol Education Program (NCEP) Step 1 guidelines (<30\% fat, <10\% saturated fat and $<300 \mathrm{mg}$ cholesterol per day). In insulin-sensitive individuals, increasing dietary cholesterol by feeding the equivalent of 4 eggs per day, but not 2 eggs per day, resulted in significant increases in TC, LDL-C, HDL-C, non-HDL-C, and apoB concentrations. This pattern was also observed in the two insulin-resistant groups, although the differences, for the most part, did not reach statistical significance. These data suggest the effects of dietary cholesterol were more prominent in insulinsensitive than insulin-resistant individuals.

Using a randomized crossover design, Herron et al. instructed 91 individuals (40 men, average age 32.6 years, and 51 women, average age 29.5 years) to consume a NCEP Step I diet [30•]. The dietary variable was 3 eggs per day or a cholesterol-free, fat-free egg substitute. The intervention period was 4 weeks per phase, and there was a 3-week washout period. The data were analyzed based on ABCG5 alleles. During the egg-supplemented period, individuals with the $\mathrm{C}$ / $\mathrm{C}$ ABCG5 allele had a significantly greater increase in plasma TC and LDL-C than either the $\mathrm{C} / \mathrm{G}$ or $\mathrm{G} / \mathrm{G}$ alleles. The NCEP
Step 1 diet resulted in a significant increase in HDL-C concentrations during both diet periods, and this was independent of ABCG5 genotype. These results suggest that the ABCG5 allele may affect an individual's response to changes in dietary cholesterol.

Chakrabarty et al. conducted a crossover design study involving 34 subjects (average age 25.7 years) who were instructed to consume a lacto-vegetarian diet and avoid baked goods (to minimize sources of dietary cholesterol) [31•]. The intervention variable was $1 \mathrm{egg}$ per day (300 mg cholesterol) or no supplemental food. Each diet phase was 8 weeks and was not separated by a washout period. There were no significant differences at the end of the diet periods for TC, LDL-C, HDL-C, or triglyceride concentrations. One egg per day resulted in a higher TC/HDL-C ratio. Considerable variability was noted among study subjects. Differences between the diet phases in LDL-C concentrations ranged from $15 \%$ to $73 \%$. A secondary analysis was conducted after dividing subjects into two groups, hyperresponders $(\mathrm{n}=12)$ and hyporesponders $(\mathrm{n}=$ 22), defined as having less than or greater than a $15 \%$ change in LDL-C concentrations in response to 1 egg per day. Compared to the no-supplemental-egg, hyperresponders, but not hyporesponders, had significantly higher TC and LDL-C concentrations and TC/HDL-C ratio. 


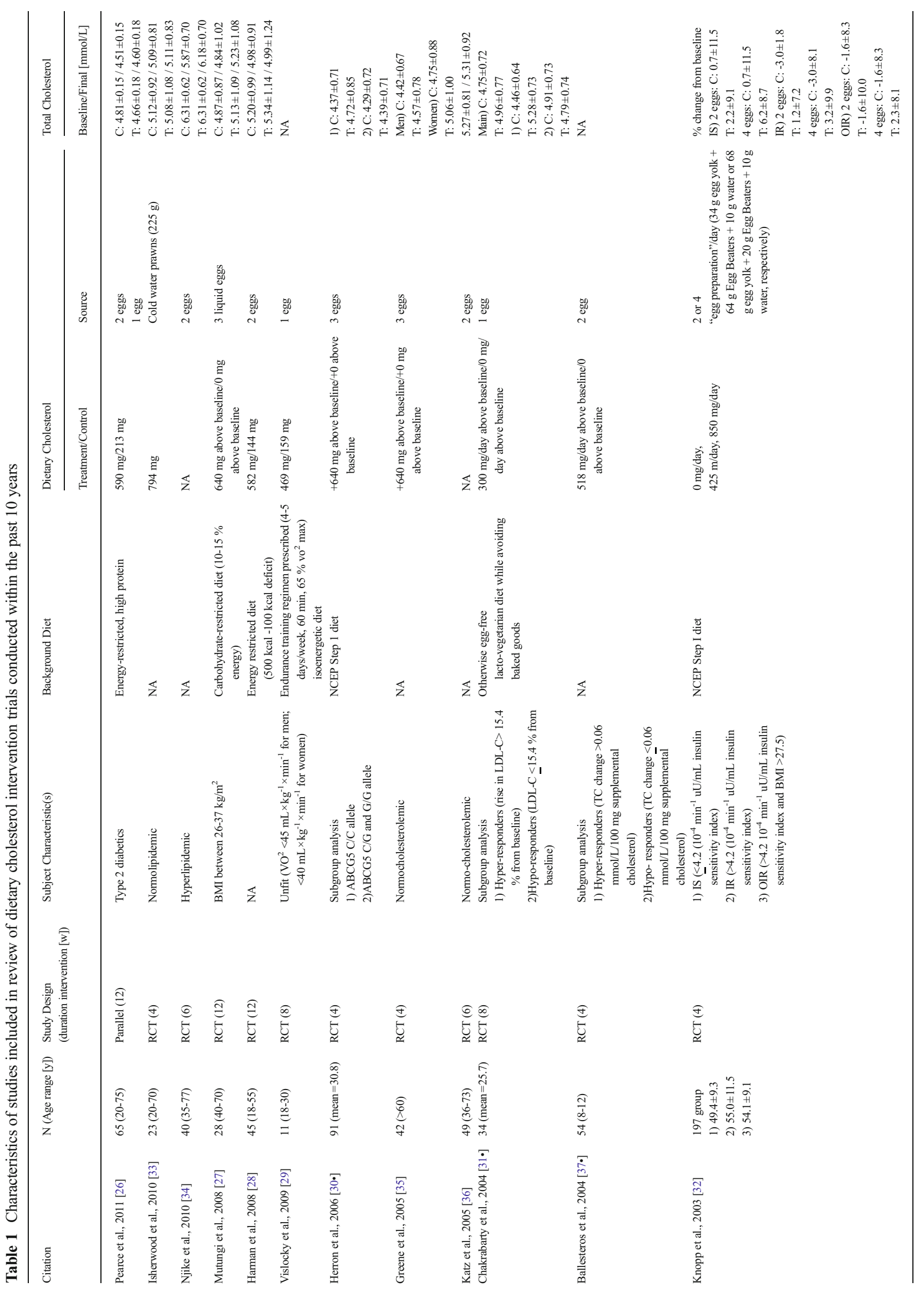




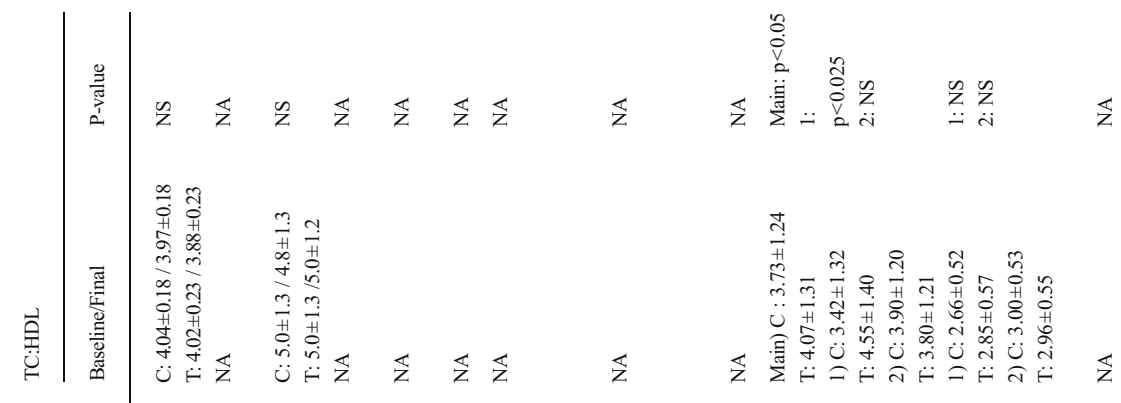

.

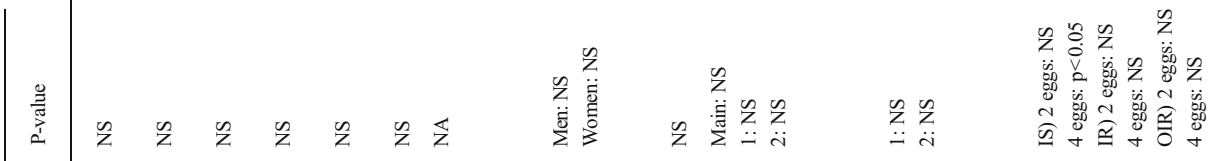

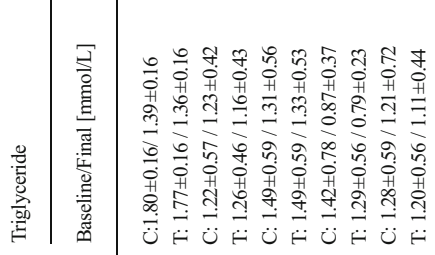

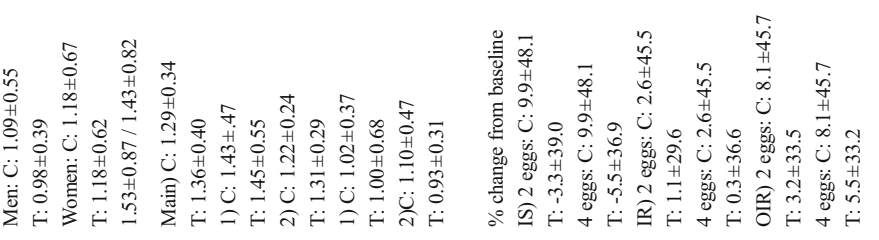

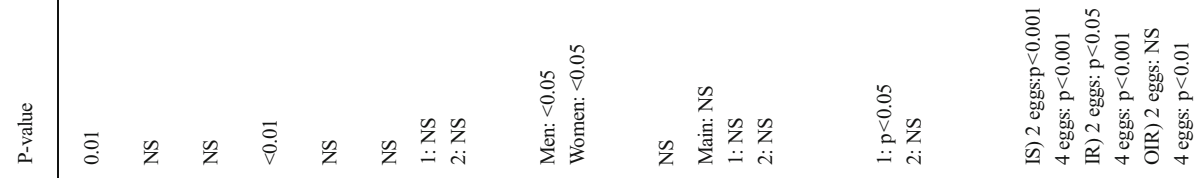

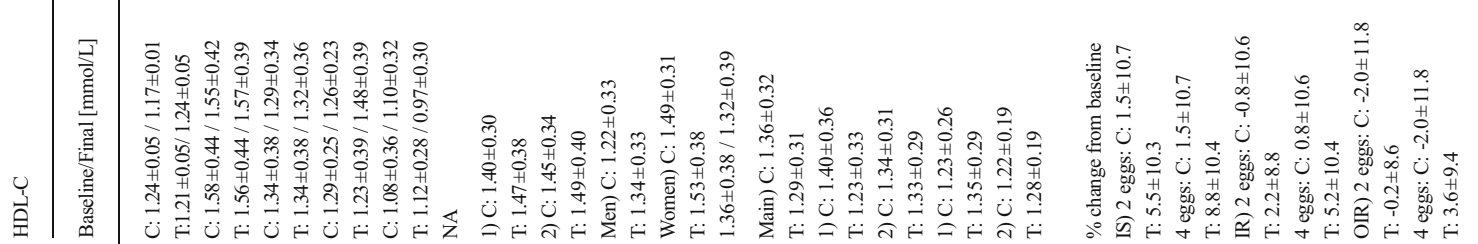

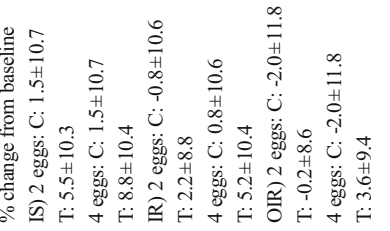

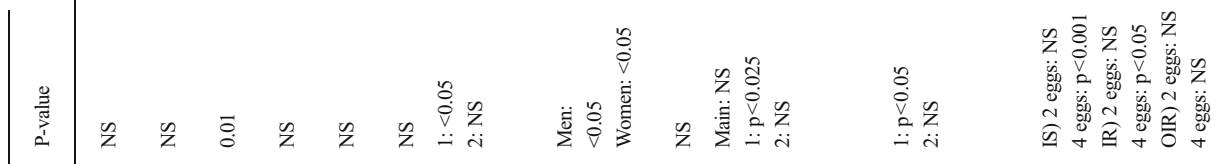

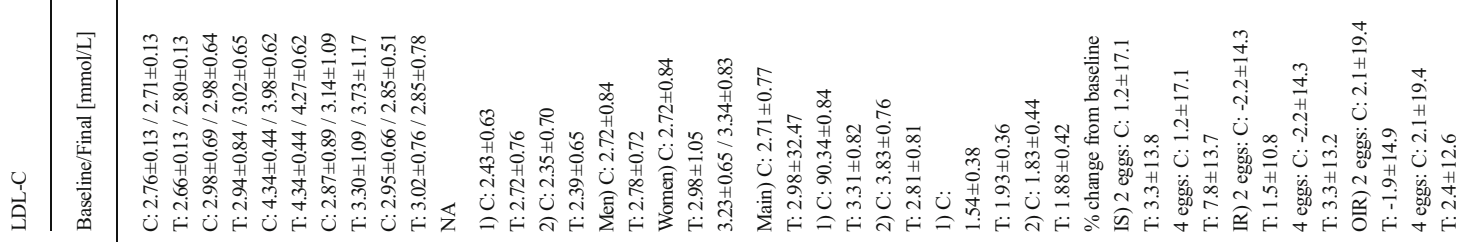

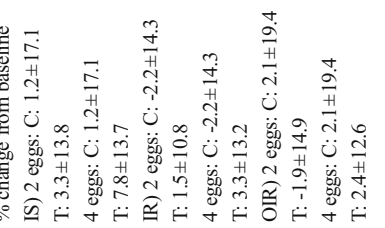

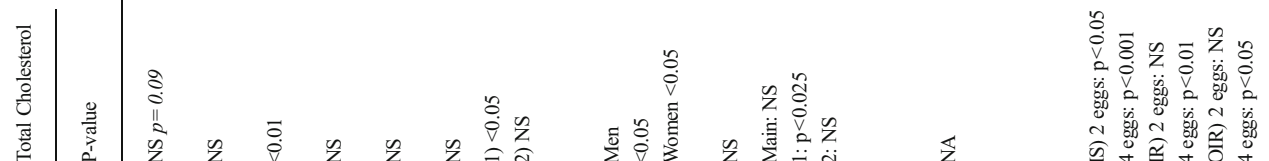


Dietary Cholesterol Supplementation Without Controlling the Background Diet

Katz et al. compared the effect of 2 eggs per day (600 mg cholesterol) or uncooked oatmeal ( $60 \mathrm{~g}, 0 \mathrm{mg}$ cholesterol) in 49 normocholesterolemic adult subjects (30 men, average age 54.1 years, and 19 women, average age 59.2 years) on plasma cholesterol concentrations and brachial artery flow-mediated dilation (FMD) using a randomized controlled crossover design [36]. Each diet phase was 6 weeks, and there was a 4-week washout phase. Compared to baseline values, the 2 supplemental eggs per day had no significant effect on TC, LDL-C, HDL$\mathrm{C}$, and triglyceride concentrations, or FMD. In contrast, $60 \mathrm{~g}$ of uncooked oats resulted in significant reductions in TC and LDL-C and no changes in HDL-C and triglyceride concentrations, or FMD, compared to baseline values.

Using a randomized crossover design, Ballesteros et al. assigned 54 children (aged 8-12 years) to add either 110 grams of eggs - the equivalent of 2 eggs per day (518 $\mathrm{mg}$ cholesterol) or an equivalent amount of cholesterol-free egg white substitute to their habitual diet [37०]. Each diet phase was 30 days, and there was a 3-week washout period. At the end of the study, the children were divided into hyporesponders and hyperresponders based on their change in plasma TC concentrations at the end of the egg diet phase: change in TC concentration from baseline less than or greater than $0.05 \mathrm{mmol} / \mathrm{L}$ per $100 \mathrm{mg}$ supplemental dietary cholesterol, respectively. Compared to baseline, the addition of 2 eggs to the diet of hyperresponders significantly increased LDL-C and HDL-C but not triglyceride and apo B concentrations or TC/HDL-C ratio. There was no significant effect of supplemental dietary cholesterol in the hyporesponders. At the end of the intervention periods, the difference in LDL-C and HDL-C concentrations between hyperresponders and hyporesponders was significant. Supplemental eggs, but not egg substitute, significantly increased mean LDL peak diameter in both hyporesponders and hyperresponders, as indicated by a reduction in the smaller LDL subfractions.

Greene et al. conducted a randomized crossover trial in 42 normocholesterolemic men and postmenopausal women under the age of 60 , comparing the effects of 3 large eggs per day (640 mg cholesterol) or an equivalent amount of cholesterolfree, fat-free egg substitute [35]. At the end of each 4-week intervention period, separated by a 3-week washout period, there was no significant effect of supplemental eggs on TC, LDL-C, and HDL-C concentrations, and no significant change in triglyceride concentration or the LDL/HDL ratio, compared to the egg-substitute period. As previously reported [37•], the supplemental eggs resulted in a significant increase in LDL peak diameter.

Isherwood et al. conducted a randomized crossover trial in 23 normocholesterolemic male subjects aged 19-67 years [33]. Subjects were provided with $225 \mathrm{~g}$ of cold-water prawns per day ( $750 \mathrm{mg}$ cholesterol) or $225 \mathrm{~g}$ of crab sticks per day ( $200 \mathrm{mg}$ cholesterol) for 4 weeks, each with a 4 -week washout period. Neither intervention resulted in a significant change in plasma TC, LDL-C, HDL-C, triglyceride, apo A1 and apo B concentrations, or LDL particle size. The authors suggested that the lack of effect may have been attributable to the characteristics of the study subjects, normocholesterolemic, or the non-cholesterol sterol content of the prawns, which may have interfered with cholesterol absorption.

A study performed by Njike and coworkers randomized 40 subjects aged 35 to 77 years and diagnosed with hyperlipidemia (as defined by serum total cholesterol $>240 \mathrm{mg} / \mathrm{dL}$ and/ or LDL cholesterol $>160 \mathrm{mg} / \mathrm{dL}$ and/or a total cholesterol/ HDL ratio $>5.7)$ to receive either two eggs $(400 \mathrm{mg}$ cholesterol) or an equivalent amount of a cholesterol-free egg substitute daily for six weeks [34]. There were no additional dietary instructions. At the end of the intervention period, there were no significant differences in plasma lipid concentrations in the egg-supplemented group. However, in the cholesterol-free egg substitute group, TC and LDL-C concentrations were significantly lower. Triglyceride concentrations compared to baseline were significantly lower in both groups. It is difficult to draw conclusions from this study due to the absence of data on how the groups receiving the eggs or egg substitute compensated with respect to the other components of the diet. The potential for no change in the dietary cholesterol intake in the group receiving eggs and an exchange of the egg substitute for habitually consumed eggs in the other group cannot be ruled out.

\section{Summary}

Among the studies that met our eligibility criteria, there was a wide range of study types, interventions, and participants. The study subjects included normolipidemic and hyperlipidemic, adults and children, normal weight and overweight/obese, and insulin-sensitive and insulin-insensitive. In some studies, the data were stratified on the basis of cholesterol responsiveness and ABCG5 genotypes. Diets administered were intended to maintain body weight or promote weight loss. Both parallel and crossover study designs were used. Intervention periods ranged from 4 to 12 weeks. In one case, diets were restricted in meat. In other cases, study subjects were instructed to follow NCEP Step 1 diet guidelines. The majority of the studies compared eggs as the source of dietary cholesterol to cholesterol-free egg substitutes.

Most studies assessed the effect of 1-3 eggs per day. The majority of studies that controlled for background diet reported either no significant effect on LDL-C concentrations [26-29] or an effect that was significant only after subgroup analysis on the basis of responsiveness or genotype [30, 32$]$. Data for the studies in which the background diet was not 
controlled were mixed for LDL-C concentrations, with 2 studies reporting no significant effect [33, 36], 1 study reporting no significant effect in hyporesponders and a significant increase in hyperresponders [37•], and 1 study reporting no significant effect in response to 2 eggs per day but a significant decrease in the group receiving the cholesterolfree egg substitute [34]. There were no trends on the basis of whether the intervention included 2 or 3 eggs per day, which represented the majority of the studies, or the length of the intervention period. Those studies that coupled weight loss with supplemental egg intake were less likely to report an effect on LDL-C concentrations. Effects of supplemental eggs on HDL-C concentrations tended to be small, albeit significant in some cases, and generally resulted in an increase. There was little effect of supplemental cholesterol on plasma triglyceride concentrations.

\section{Conclusions}

Over the past 10 years, there have been only a limited number of studies on the effect of dietary cholesterol on plasma cholesterol concentrations. Striking among the available studies is the high degree of variability in background diet, study subject characteristics, and study design. Within the context of current levels of dietary cholesterol, the effect on plasma lipids concentrations, with primary interest in LDL-C concentrations, is modest and appears to be limited to population subgroups. In these cases, restrictions in dietary cholesterol intake are likely warranted. The biological determinants of interindividual variability remain a relatively understudied area.

\section{Compliance with Ethics Guidelines}

Conflict of Interest John D. Griffin declares that he has no conflict of interest.

Alice H. Lichtenstein declares that she has no conflict of interest.

Human and Animal Rights and Informed Consent This article does not contain any studies with human or animal subjects performed by any of the authors.

\section{References}

Papers of particular interest, published recently, have been highlighted as:

- Of importance

1. Go AS, Mozaffarian D, Roger VL, Benjamin EJ, Berry JD, Borden WB, Bravata DM, Dai S, Ford ES, Fox CS, et al. Executive summary: heart disease and stroke statistics -2013 update: a report from the American Heart Association. Circulation. 2013;127:143-52.
2. Expert Panel on Detection Evaluation and Treatment of High Blood Cholesterol in Adults (Adult Treatment Panel III). Executive summary of the third report of the National Cholesterol Education Program (NCEP). J Am Med Assoc. 2001;285:2486-97.

3. Finking G, Hanke H. Nikolaj Nikolajewitsch Anitschkow (18851964) established the cholesterol-fed rabbit as a model for atherosclerosis research. Atherosclerosis. 1997;135:1-7.

4. IOM. Dietary reference intakes for energy, carbohydrate, fiber, fat, fatty acids, cholesterol, protein and amino acids. Washington, DC: National Academy of Sciences; 2005. p. 482.

5. Lichtenstein AH, Appel LJ, Brands M, Carnethon M, Daniels S, Franch HA, et al. Diet and lifestyle recommendations revision 2006: a scientific statement from the American Heart Association Nutrition Committee. Circulation. 2006;114:82-96.

6. Dietary Guidelines for Americans: http://www.cnpp.usda.gov/ DGAs2010-DGACReport.htm. 2010.

7. Lecerf J-M, de Lorgeril M. Dietary cholesterol: from physiology to cardiovascular risk. Br J Nutr. 2011;106:6-14.

8. van der Wulp MY, Verkade HJ, Groen AK. Regulation of cholesterol homeostasis. Mol Cell Endocrinol. 2013;368:1-16.

9. Abumrad NA, Davidson NO. Role of the gut in lipid homeostasis. Physiol Rev. 2012;92:1061-85.

10. Grande F, Anderson J, Chlouverakis C, Proja M, Keys A. Effect of dietary cholesterol on man's serum lipids. J Nutr. 1965;87:52-62.

11. Clarke R, Frost C, Collins R, Appleby P, Peto R. Dietary lipids and blood cholesterol: quantitative meta-analysis of metabolic ward studies. Br Med J. 1997;314(7074):112-7.

12. Howell W, McNamara D, Tosca M, Smith B, Gaines J. Plasma lipid and lipoprotein responses to dietary fat and cholesterol: a metaanalysis. Am J Clin Nutr. 1997;65:1747-64.

13. Weggemans R, Zock P, Katan M. Dietary cholesterol from eggs increases the ratio of total cholesterol to high-density lipoprotein cholesterol in humans: a meta-analysis. Am J Clin Nutr. 2001;73:885-91.

14. Food and Nutrition Board and American Heart Association. Diet and coronary heart disease. Nutr Rev. 1972;30(10):223-5.

15. AHA. Dietary guidelines for healthy American adults. Circulation. 1986;74:1465A-8A.

16. Expert Panel on Detection E, and Treatment of High Blood Cholesterol in Adults. Report of the National Cholesterol Education Program Expert Panel on detection, evaluation, and treatment of high blood cholesterol in adults. Arch Intern Med. 1988;148(1):36-69.

17. Expert Panel on Detection E, and Treatment of High Blood Cholesterol in Adults. Summary of the second report of the National Cholesterol Education Program (NCEP) expert panel on detection, evaluation, and treatment of high blood cholesterol in adults (adult treatment panel II). J Am Med Assoc. 1993;269(23): 3015-23.

18. Krauss RM, Deckelbaum RJ, Ernst N, Fisher E, Howard BV, Knopp $\mathrm{RH}$, et al. Dietary guidelines for healthy American adults. A statement for health professionals from the Nutrition Committee, American Heart Association. Circulation. 1996;94(7):1795-800.

19. Krauss RM, Eckel RH, Howard B, Appel LJ, Daniels SR, Deckelbaum RJ, et al. AHA dietary guidelines: revision 2000: a statement for healthcare professionals from the Nutrition Committee of the American Heart Association. Circulation. 2000;102(18):2284-99.

20. Djousse L, Gaziano J. Egg consumption in relation to cardiovascular disease and mortality: the Physicians' Health Study. Am J Clin Nutr. 2008;87:964-9.

21. Rong Y, Chen L, Zhu T, Song Y, Yu M, Shan Z, et al. Egg consumption and risk of coronary heart disease and stroke: dose response metaanalysis of prospective cohort studies. Br Med J. 2013;346:e8539.

22. Shin JY, Xun P, Nakamura Y, He K. Egg consumption in relation to risk of cardiovascular disease and diabetes: a systematic review and meta-analysis. Am J Clin Nutr. 2013;98:146-59. 
23. Katan MB, Beynen AC, de Vries JH, Nobels A. Existence of consistent hypo- and hyperresponders to dietary cholesterol in man. Am J Epidemiol. 1986;123(2):221-34.

24. Rudkowska I, Jones PJH. Polymorphisms in ABCG5/G8 transporters linked to hypercholesterolemia and gallstone disease. Nutr Rev. 2008;66:343-8.

25. Sources of dietary cholesterol: http://riskfactor.cancer.gov/diet/ foodsources/cholesterol/table1.html. 2013.

26. Pearce K, Clifton P, Noakes M. Egg consumption as part of an energy-restricted high-protein diet improves blood lipid and blood glucose profiles in individuals with type 2 diabetes. Br J Nutr. 2011;105:584-92.

27. Mutungi G, Ratliff J, Puglisi M, Torres-Gonzalez M, Vaishnav U, Leite J, et al. Dietary cholesterol from eggs increases plasma HDL cholesterol in overweight men consuming a carbohydrate-restricted diet. J Nutr. 2008;138:272-6.

28. Harman N, Leeds A, Griffin B. Increased dietary cholesterol does not increase plasma low density lipoprotein when accompanied by an energy-restricted diet and weight loss. Eur J Nutr. 2008;47:287-93.

29. Vislocky L, Pikosky M, Rubin K, Vega-López S, Gaine P, Martin W, et al. Habitual consumption of eggs does not alter the beneficial effects of endurance training on plasma lipids and lipoprotein metabolism in untrained men and women. J Nutr Biochem. 2009;20:26-34.

30. - Herron K, McGrane M, Waters D, Lofgren I, Clark R, Ordovas J, et al. The ABCG5 polymorphism contributes to individual responses to dietary cholesterol and carotenoids in eggs. J Nutr. 2006;136:1161-5. This article assessed the effect of a relatively large cholesterol challenge, 3 eggs per day, on the basis of ABCG5 polymorphisms.
31. - Chakrabarty G, Bijlani R, Mahapatra S, Mehta N, Lakshmy R, Vashisht $\mathrm{S}$, et al. The effect of ingestion of egg on serum lipid profile in healthy young free-living subjects. Indian J Physiol Pharmacol. 2004;46:492-8. This article assessed the effect of a modest cholesterol challenge, 1 egg per day, within the context of hyper and hyporesponder adults.

32. Knopp R, Retzlaff B, Fish B, Walden C, Wallick S, Anderson M, et al. Effects of insulin resistance and obesity on lipoproteins and sensitivity to egg feeding. Arterioscler Thromb Vasc Biol. 2003;23:1437-43.

33. Isherwood C, Wong C, Jones W, Davies I, Griffin B. Lack of effect of cold water prawns on plasma cholesterol and lipoproteins in normolipidaemic men. Cell Mol Biol. 2010;56:52-8.

34. Njike V, Faridi Z, Dutta S, Gonzalez-Simon AL, Katz DL. Daily egg consumption in hyperlipidemic adults-effects on endothelial function and cardiovascular risk. Nutr J. 2010;9:28.

35. Greene C, Zern T, Wood R, Shrestha S, Aggarwal D, Sharman M, et al. Maintenance of the LDL cholesterol: HDL cholesterol ratio in an elderly population given a dietary cholesterol challenge. J Nutr. 2005; 135:2793-8.

36. Katz D, Evans M, Nawaz H, Yanchou Njike V, Chan W, Comerford B, et al. Egg consumption and endothelial function: a randomized controlled crossover trial. Int J Cardiol. 2005;99:65-70.

37. • Ballaesteros M, Cabrera R, del Socorro Saucedo M, Luz Fernandez M. Dietary cholesterol does not increase biomarkers for chronic disease in a pediatric population from northern Mexico. Am J Clin Nutr. 2004;80:855-61. This article assessed the effect of a moderate cholesterol challenge, 2 eggs per day, within the context of hyper and hypo-responder children. 